\title{
FINITE GROUPS WITH SMALL AUTOMIZERS OF THEIR NONABELIAN SUBGROUPS
}

\author{
ROLF BRANDL \\ Mathematisches Institut der Universität, Am Hubland 12, D-97074 Würzburg, Germany \\ MARIAN DEACONESCU \\ Department of Mathematics, Kuwait University, P.O. Box 5969, Safat 13060, Kuwait
}

(Received 25 February, 1997)

1. Introduction. Let $G$ be a group and let $H$ be a subgroup of $G$. The automizer $\operatorname{Aut}_{G}(H)$ of $H$ in $G$ is defined as the group of automorphisms of $H$ induced by conjugation of elements of $N_{G}(H)$. Thus $\operatorname{Aut}_{G}(H) \cong N_{G}(H) / C_{G}(H)$, and we obviously have

$$
\operatorname{In}(H) \leq \operatorname{Aut}_{G}(H) \leq \operatorname{Aut}(H) .
$$

We call Aut $A_{G}(H)$ large if $\operatorname{Aut}_{G}(H)=\operatorname{Aut}(H)$ and small if $\operatorname{Aut}_{G}(H)=\operatorname{In}(H)$.

$\mathrm{H}$. Zassenhaus proved in [10] an elegant result which can be rephrased as follows: a finite group is abelian if and only if the automizers of all its abelian subgroups are small. Lennox and Wiegold [6] have considered groups, in which the automizers of all subgroups are large, that they called MD-groups. The finite MDgroups are scarce: they are isomorphic to the symmetric groups $S_{n}$ for $n \leq 3$ - see also Deaconescu [2]. For more about infinite MD-groups, see [7] and [8].

Here we consider finite nonabelian groups in which the automizers of all nonabelian subgroups are small; for convenience, we shall call these groups SANSgroups (Small Automizers for Nonabelian Subgroups). By definition, this is equivalent to saying that $N_{G}(H)$ induces inner automorphisms on every nonabelian subgroup $H$ of $G$. The latter condition is equivalent to $N_{G}(H)=H C_{G}(H)$, for every nonabelian subgroup $H$ of $G$. These conditions will frequently be used instead of the original definition without further reference.

The finite minimal nonabelian groups, that is the finite nonabelian groups all of whose proper subgroups are abelian, are typical examples of SANS-groups. All these are soluble SANS-groups, but there exist simple SANS-groups as well, as for example $L_{2}(5)$. This raises the problem of describing the structure of SANS-groups. Our main result is as follows.

TheOREM. I. A p-group $G$ is a SANS-group if and only if $G=T Z(G)$, where $T$ is a minimal nonabelian subgroup of $G$. In particular, if $G$ is a $S A N S$-group, then $\left|G^{\prime}\right|=p$ and $|G: Z(G)|=p^{2}$.

II. If a nilpotent group $G$ with $|\pi(G)| \geq 2$ is a $S A N S$-group, then $G=P \times O_{p^{\prime}}(G)$, where $P \in \operatorname{Syl}_{p}(G)$ is a $S A N S$-group and $O_{p^{\prime}}(G)$ is abelian.

III. If $G$ is a soluble nonnilpotent SANS-group, then $F(G)$ is abelian and $|G: F(G)|$ is a prime.

IV. If $G$ is a nonsoluble SANS-group, then $G=H \times Z(G)$. Here $H$ is a simple group isomorphic to $L_{2}(q)$, where $q=2^{n}$, where $n \geq 3$ and $q-1$ is a Mersenne prime, or $q$ is odd and $(q-1) / 2$ is a prime. 
Since the structure of the minimal nonabelian p-groups is known ([4, p. 309]), the Cases I and II of the Theorem give a complete description of the nilpotent SANS-groups. It will be shown that the condition in Case III is a sufficient one, provided that all Sylow subgroups of $G$ are abelian.

The notation is standard and follows that of Aschbacher [1] and Huppert [4]. All groups considered here are finite.

2. Preliminaries. We need the following result of Zassenhaus [10]. The original proof is somewhat lengthy and so the following simple argument may be of some interest.

Lemma 2.1. Let $G$ be a group in which $N_{G}(H)=C_{G}(H)$, for every abelian subgroup $H$ of $G$. Then $G$ is abelian.

Proof. Let $P \in \operatorname{Syl}_{p}(G)$ and let $A$ be a maximal abelian normal subgroup of $P$. Then $A=C_{P}(A)$, and since, by hypothesis, $P$ induces in $A$ only inner automorphisms, one obtains $P=A C_{P}(A)=A$. Hence $P$ is abelian.

The hypothesis, applied to $P$, yields $N_{G}(P)=C_{G}(P)$ and, by a well-known result of Burnside ([4, Hauptsatz 2.6, p. 419]), $G$ is $p$-nilpotent. As this is true for all primes, $G$ is nilpotent and the result follows.

The proof of the theorem relies on the following two elementary lemmas.

Lemma 2.2. Every nonabelian section of a SANS-group $G$ is a SANS-group. In particular, $G$ does not have any section isomorphic to the symmetric group $S_{4}$.

Proof. The proof of this lemma is straightforward and we omit it.

Lemma 2.3. Let $G=A \times B$ be a SANS-group. Suppose that $A$ is nonabelian. Then $B$ is abelian.

Proof. By Lemma 2.1, it suffices to prove that if $T$ is an abelian subgroup of $B$ then $N_{B}(T)=C_{B}(T)$.

By way of contradiction, assume that there exists $b \in N_{B}(T) \backslash C_{B}(T)$. Consider the nonabelian subgroup $H=A \times T$ of $G$. Then $b \in N_{G}(H)$. Moreover, we have $C_{G}(H)=Z(A) \times C_{B}(T)$, which implies $H C_{G}(H)=A \times C_{G}(B)$. Thus $b \notin H C_{G}(H)$ and $N_{G}(H) \neq H C_{G}(H)$, a contradiction.

The next result provides a class of examples of soluble, nonnilpotent SANSgroups.

Proposition 2.4. Let $G$ be a group with abelian Sylow subgroups. Suppose that $|G: F(G)|=p$, where $p$ is a prime. Then $G$ is a SANS-group.

Proof. By hypothesis, $F(G)$ is abelian, and so we can apply a result of Taunt (see $\left[4\right.$, p. 752]) in order to deduce that $F(G)=G^{\prime} \times Z(G)$.

Let $H$ be a nonabelian subgroup of $G$. First, assume that $H$ is normal in $G$. We need to show $H C_{G}(H)=G$. As $H$ is nonabelian, we have $H \nsubseteq F(G)$. Let $x \in H \backslash F(G)$. Since $|G: F(G)|=p$, we get $G=F(G)\langle x\rangle$. First of all, note that $G^{\prime}=\left\langle x^{G}\right\rangle \leq H$ as $H$ is normal in $G$. As clearly $Z(G) \leq C_{G}(H)$, this implies that 


$$
H C_{G}(H) \geq\left\langle G^{\prime}, Z(G), x\right\rangle=\langle F(G), x\rangle=G,
$$

as claimed.

If $H$ is nonnormal in $G$, the argument above, applied within $N_{G}(H)$, proves that $N_{G}(H)=H C_{G}(H)$. Thus $G$ is a SANS-group and the proof is complete.

Finally, we record a series of simple SANS-groups.

Lemma 2.5. (i) The groups $S L_{2}(q)$, with q odd, are not $S A N S$-groups.

(ii) Let $G=L_{2}\left(2^{n}\right)$, where $n \geq 3$. Then $G$ is a SANS-group if and only if $2^{n}-1$ is a prime.

(iii) Let $G=P S L(2, q)$, where $q=p^{f}$ is odd and $q \geq 5$. Then $G$ is a SANS-group if and only if $(q-1) / 2$ is a prime. In particular, either $q=3^{f}$, where $f$ is a prime, or $q=p \geq 5$ is a prime.

Proof. (i) Let $G=S L_{2}(q)$, with $q$ odd. Then $G$ contains precisely one involution $z$ and $Z(G)=\langle z\rangle$. By Dickson's list [4, p. 213], the group $G / Z(G)$ contains a subgroup $K / Z(G)$ isomorphic to $A_{4}$. Its preimage $K$ is nonabelian and $K$ has a Sylow 2-subgroup $H$ isomorphic to the quaternion group $Q_{8}$. In particular, $H$ is nonabelian. But the elements of order 3 of $K$ act as outer automorphisms on $H$, which proves that $G$ is not a SANS-group.

(ii) Let $G=L_{2}\left(2^{n}\right)$, where $n \geq 3$, and suppose that $2^{n}-1=a b(a, b>1)$ is not a Mersenne prime. The normalizer of a Sylow 2-subgroup of $G$ has a nonabelian normal subgroup of order $\left(2^{n}-1\right) a$ on which a cyclic group of order $b$ acts as a group of outer automorphisms. Thus $G$ is not a SANS-group. The converse follows from an inspection of Dickson's list.

(iii) The first statement follows as in (ii), while the remaining ones are obvious using elementary number theory.

3. Proof of the theorem. We split the proof into several cases, according to whether $G$ is a $p$-group, a nilpotent group, a soluble nonnilpotent group and a nonsoluble group.

\section{The p-group $G$ is a SANS-group.}

If $G$ is a minimal nonabelian group the result follows from a theorem of Rédei quoted in [4, p. 309]. Let now $M$ be a nonabelian maximal subgroup of $G$. Then we have $G=N_{G}(M)=M C_{G}(M)$. But $Z(M)=M \cap C_{G}(M) \leqslant Z(G)$ and, since $|G: M|=\left|C_{G}(M): Z(M)\right|=p$, this implies that $C_{G}(M)$ is abelian. Thus $G=M C_{G}(M) \leqslant C_{G}\left(C_{G}(M)\right)$, forcing $C_{G}(M)=Z(G)$. Hence $G=M Z(G)$.

By Lemma 2.2 and by induction on the order of $G$, we have $M=T Z(M)$, where $T$ is a minimal nonabelian subgroup of $M$. Thus $G=M Z(G)=T Z(M) Z(G)=T Z(G)$. Since by Rédei's result $p=\left|T^{\prime}\right|=\left|G^{\prime}\right|$ and $p^{2}=|T: Z(T)|=|G: Z(G)|$, the necessity is proved.

To prove sufficiency, let $G$ be a $p$-group and suppose that $G$ has a minimal nonabelian subgroup $T$ such that $G=T Z(G)$. Let $K$ be a nonabelian subgroup of $G$. In order to show that $G$ is a SANS-group, we have to prove that $N_{G}(K)=K C_{G}(K)$.

First, as $K$ is nonabelian and $\left|G^{\prime}\right|=p$, we have $G^{\prime}=K^{\prime}$, and so $K$ is normal in $G$. We thus need to show that $G=K C_{G}(K)$. For this, we note that 
$K C_{G}(K) / C_{G}(K) \cong K / Z(K)$ is of order at least $p^{2}$ as $K$ is nonabelian. But $Z(K) \leqslant C_{G}(K) \leqslant K C_{G}(K) \leqslant G$ and $|G: Z(G)|=p^{2}$. The remarks above yield $G=K C_{G}(K)$. Thus $G$ is a SANS-group and the proof is complete.

II. $G$ is a nilpotent $S A N S$-group and $|\pi(G)| \geq 2$.

In this case, since $G$ is nonabelian by definition, there exists a nonabelian Sylow $p$-subgroup $P$ of $G$ and, of course, we have $G=P \times O_{p^{\prime}}(G)$. Lemma 2.2 implies that $P$ is a SANS-group, and by Lemma 2.3 we get that $O_{p^{\prime}}(G)$ must be abelian.

\section{III. $G$ is a soluble, nonnilpotent SANS-group.}

If $F(G)$ is nonabelian, then $G=N_{G}(F(G))=F(G) C_{G}(F(G))=F(G)$, contrary to the hypothesis. Therefore $F(G)$ is abelian. If $H / F(G)$ is a nonabelian minimal subgroup of $G / F(G)$, then clearly $F(G)<H$, and $H$ is nonabelian since $H$ is nonnilpotent. Thus $C_{G}(H) \leqslant C_{G}(F(G)) \leqslant F(G)<H$, whence $G=N_{G}(H)=$ $H C_{G}(H) \leqslant H$. Since $G=H$, the solvability of $G$ implies that $|G: F(G)|$ is a prime.

We remark that Proposition 2.4 provides a partial converse to the statement of Case III, provided that all Sylow subgroups of $G$ are abelian.

IV. G is a nonsoluble SANS-group.

Let $G$ denote for the moment an arbitrary finite nonsoluble group. Recall that a component of $G$ is a subnormal subgroup $X$ of $G$ such that $X^{\prime}=X$ and $X / Z(X)$ is a simple group. If $E(G)=\langle X| X$ is a component of $G\rangle$, the generalized Fitting subgroup of $G$ is the subgroup $F^{*}(G)=E(G) F(G)$. The next lemma summarizes some of the main properties of $E(G)$ and $F^{*}(G)$.

Lemma 3.1. (i) $F^{*}(G)$ is a central product of $E(G)$ and $F(G)$. Moreover, we have $C_{G}(F *(G)) \leqslant F^{*}(G)$.

(ii) $E(G) / Z(E(G))$ is a direct product of simple nonabelian groups; the number of factors in the direct product is equal to the number of components of $G$.

(iii) If $S / Z(F(G))$ is the socle of $C_{G}(F(G)) / Z(F(G))$, then $S=E(G) Z(F(G))$ and $E(G)=E(G)^{\prime}=S^{\prime}$.

(iv) $F\left(C_{G}(F(G)) / Z(F(G))=1\right.$.

Proof. See Chapter 11 of [1].

From now on $G$ will denote a nonsoluble SANS-group. The next two lemmas are essential.

Lemma 3.2. (i) Every chief factor of $\mathrm{G}$ is either abelian or simple.

(ii) If $1<H \triangleleft G$ and if $Z(H)=1$, then $G=H \times Z(G)$.

Proof. (i) This follows from Lemmas 2.1 and 2.3.

(ii) As $H$ is nonabelian and $H \cap C_{G}(H)=Z(H)=1$ we get $G=N_{G}(H)=$ $H \times C_{G}(H)$. By Lemma 2.3, we know that $C_{G}(H)$ is abelian and so $C_{G}(H)=Z(G)$. 
Lemma 3.3. Suppose that $G$ is nonsimple. Then

(i) $F(G) \neq 1$.

(ii) $G=F^{*}(G)=E(G) F(G)$.

(iii) $G$ has a unique component.

Proof. (i) Suppose that $F(G)=1$. Since $G$ is nonsimple and nonsoluble, $G$ has a minimal nonabelian normal subgroup $H$ and, by Lemma 3.2(ii), $G=H \times Z(G)$. This contradicts $F(G)=1$.

(ii) Since $G$ is nonsoluble, $E(G)$ is nontrivial and $F^{*}(G)=E(G) F(G)$ is nonabelian. Thus $G=N_{G}\left(F^{*}(G)\right)=F^{*}(G) C_{G}\left(F^{*}(G)\right)=F^{*}(G)$, by Lemma 3.1(i).

(iii) By hypothesis and by Lemmas 2.2, 2.3 and 3.1(ii), we get that $E(G) / Z(E(G))$ is a nonabelian simple group.

At this stage we split our discussion into four subcases.

IV(a). $G$ is a simple group.

By Lemma 2.2, no section of $G$ is isomorphic to $S_{4}$. Hence a result of Glauberman (see [3, Theorem 7.1]) implies that $G$ is one of the following groups.

(i) A simple group of Ree type.

(ii) $S z\left(2^{2 n+1}\right)$, with $n \geq 1$.

(iii) $U_{3}\left(2^{n}\right)$, with $n \geq 2$.

(iv) The first Janko group $J_{1}$.

(v) $L_{2}(q)$, where either $q=2^{n}$, with $n \geq 3$, or $q \geq 5$ and $q \equiv 3,5(\bmod 8)$.

If $G$ is of Ree type, then Theorem 13.2 of [5] implies that $G$ has a 3-subgroup $P$ with $\left|P^{\prime}\right|>3$, contradicting Lemma 2.2 and Case I of the Theorem.

If $G$ is a Suzuki group as in (ii), then $G$ has a 2-subgroup $S$ with $\left|S^{\prime}\right|>2$, giving a contradiction as before.

If $G=U_{3}\left(2^{n}\right)$, with $n \geq 2$, then by [4, p. 242], $G$ has a 2-subgroup $Q$ with $\left|Q^{\prime}\right|>$ 2 , giving a contradiction.

If $G=J_{1}$, then $G$ has a subgroup which is a direct product of two dihedral groups of orders 6 and 10; see [9, p. 213]. But this contradicts Lemmas 2.2 and 2.3.

If $G$ is a simple SANS-group, it follows that $G=L_{2}(q)$ is as described in (v). Thus Lemma 2.5 completes the analysis of Case IV(a).

IV(b). $G$ is nonsimple and has a minimal nonabelian normal subgroup.

Let $H$ be a minimal nonabelian normal subgroup of $G$. Then $H$ is simple, by Lemma 3.2(i), while Lemma 3.2(ii) implies that $G=H \times Z(G)$. abelian.

$\mathrm{IV}(\mathrm{c}) . G$ is nonsimple; all minimal normal subgroups of $G$ are abelian and $F(G)$ is

We wish to apply part (iii) of Lemma 3.1. For this, we first determine $S$. Since $Z(F(G))=F(G)$, we have $S=E(G) F(G)=G$, by Lemma 3.3(ii). Hence part (iii) of Lemma 3.1 implies $C_{G}(F(G))=G$, and we get $F(G)=Z(G)$.

Now consider $G / Z(G)$. By Lemma 3.3(ii), we have $G=E(G) Z(G)$, so that $G / Z(G) \cong E(G) / E(G) \cap Z(G)$. Clearly, we have $Z(E(G)) \leqslant Z(G)$, so that we get $E(G) \cap Z(G)=Z(G)$. This, together with Lemma 3.3(iii), implies that $G / Z(G) \cong E(G) / Z(E(G))$ is a nonabelian simple group. 
Next consider the normal subgroup $G^{\prime} Z(G) / Z(G)$ of the simple group $G / Z(G)$. We must have $G^{\prime} Z(G)=G$. This implies $G / Z(G) \cong G^{\prime} / G^{\prime} \cap Z(G)$, which is isomorphic to one of the simple groups $Y$ listed in part IV of the Theorem. If $Y \cong L_{2}\left(2^{n}\right)$ with $n \geq 3$, then the Schur multiplicator of $Y$ is trivial [1, p. 251] and we get $G^{\prime} \cap Z(G)=1$, so that $G=G^{\prime} \times Z(G) \cong Y \times Z(G)$.

If $Y \cong L_{2}(q)$, where $q$ is odd, and if $G^{\prime} \cap Z(G) \neq 1$, it follows that $G \cong S L(2, q)$. But by Lemma 2.5(i) $G$ is not a SANS-group, a contradiction.

IV(d). $G$ is nonsimple; all minimal normal subgroups of $G$ are abelian and $F(G)$ is nonabelian.

Lemmas 3.1 and 3.3 imply $G=F^{*}(G)=F(G) E(G)$ and $[F(G), E(G)]=1$. Suppose that $F(G)$ is nonabelian. Then we cannot have $G=F(G) \times E(G)$, since both factors are nonabelian and Lemma 2.3 applies.

Thus we have $F(G) \cap E(G)=Z(E(G)) \neq 1$. In particular, the quasisimple group $E(G)$ is not simple. An inspection of the Schur multiplicators of the simple SANSgroups shows that $E(G) / Z(E(G)) \cong L_{2}(q)$, where $q$ is odd. From this, we readily get $E(G) \cong S L(2, q)$. This contradicts Lemma 2.5. The proof of the Theorem is now complete.

AcKnowledgement. The research of this author was supported by Grant SM097 from Kuwait University.

\section{REFERENCES}

1. M. Aschbacher, Finite group theory (Cambridge University Press, 1986).

2. M. Deaconescu, Problem 10270, American Mathematical Monthly 99 (1992), 958.

3. G. Glauberman, Factorizations in local subgroups of finite groups, Regional Conf. Series in Maths. No 33 (A.M.S., Providence, R.I., 1977).

4. B. Huppert, Endliche Gruppen I (Springer-Verlag, 1967).

5. B. Huppert and N. Blackburn, Finite groups II (Springer-Verlag, 1982).

6. J. Lennox and J. Wiegold, On a question of Deaconescu about automorphisms, Rend. Sem. Mat. Univ. Padova 89 (1993), 83-86.

7. A. N. Obraztsov, On a question of Deaconescu III, Rend. Sem. Mat. Univ. Padova 99 (1998), 45-82.

8. H. Smith and J. Wiegold, On a question of Deaconescu II, Rend. Sem. Mat. Univ. Padova 91 (1994), 61-64.

9. S. A. Syskin, Abstract properties of simple sporadic groups, Russian Math. Surveys 35:5 (1980), 209-246.

10. H. Zassenhaus, A group theoretic proof of a theorem of MacLagan-Wedderburn, Proc. Glasgow Math. Assoc. 1 (1952), 53-63. 